\title{
THE IMPACT OF NEW TECHNOLOGIES ON RADIOTHERAPHY
}

\author{
ROBERTO ORECCHIA *
}

ABSTRACT. - Radiation therapy has been officially recognised as an efficient cancer treatment at the International Oncology Congress in Paris on 1922, 25 years after the discovery of X-rays by W. Roentgen and since then tremendous progresses have been made on the basic knowledge on the biological effects of radiations on tumours and healthy tissues, on the modalities and times for dose delivery, planning and irradiation techniques. It may be used as the primary therapy or combined with surgical or medical therapies or to prevent serious symptoms related to the progression of the disease.

In recent years the availability of new technologies and results coming from interdisciplinary research where medical and biological competences are working in strict collaboration with physicists and engineers have contributed to a substantial improvement of this treatment by introducing new concepts and procedures which are presented in this paper.

The planning treatment has been revolutionised by the possibility to identify in quantitative way tumours and adjacent normal structures in 3D by image processing of CT and/or MRI and/or PET and using virtual simulation for more accurate placement of radiation beams. An enhancement of this technique is $\mathrm{f}$-Dimensional Conformal Radiotherapy (3DCRT) in which the profile of radiation beam is shaped to fit the profile of the target using a multileaf collimator. In radiosurgery and stereotactic radiotherapy the needed accuracy requires special new techniques to better control the uncertainty related to internal organ motion (for example due to respiration) with real-time adjustment of the radiation beam. The latest developments of Radioisotope Therapy Intra Operative Radiation Therapy and Hadrontherapy are also illustrated.

* Divisione di Radioterapia, Istituto Europeo di Oncologia e Università degli Studi di Milano. 


\section{INTRODUZIONE}

La radioterapia, nasce ufficialmente quale specialità clinica autonoma nel 1922, più di 25 anni dopo la scoperta dei raggi $\mathrm{X}$ da parte di W. Roentgen (1895) e della radioattività naturale del radium da parte di Marie e Pierre Curie (1896), a Parigi, dove, al Congresso Internazionale di Oncologia, furono presentate i primi risultati del suo impiego nel trattamento di differenti neoplasie. Da allora sono trascorsi altri 80 anni, ed in questo lasso di tempo si è verificata una enorme quantità di progressi nel campo delle conoscenze degli effetti biologici delle radiazioni sui tumori e sui tessuti sani, delle modalità e dei tempi di somministrazione della dose, della pianificazione e delle tecniche di irradiazione, della disponibilità di nuove tecnologie.

Oggi il ruolo della radioterapia è universalmente riconosciuto come rivolto alla cura del paziente affetto da tumore (occasionalmente anche in qualche caso di malattia benigna), inteso sia come trattamento radicale, esclusivo, sia in combinazione con le terapie chirurgiche e mediche, sia, ancora, con intento palliativo o per la prevenzione dei sintomi legati allo sviluppo della malattia neoplastica quando questa ha raggiunto uno stadio incompatibile con la possibilità di guarigione.

Questo risultato è ottenibile somministrando una dose ben definita di radiazioni, espressa in un'unità di misura convenzionale, il Gray (Gy), ad un determinato volume bersaglio, riducendo, per quanto possibile, l'esposizione dei tessuti e degli organi circostanti. Il bersaglio è in genere rappresentato dalla massa tumorale, come appare dalle moderne tecniche di imaging (GTV, gross tumor volume), e comprendere anche le regioni potenzialmente a rischio per diffusione microscopica della malattia (CTV, clinical target volume). A questo, occorre aggiungere un margine (PTV, planning target volume), la cui definizione è in rapporto alla caratteristiche tecniche del trattamento e ad altri fattori, tra i quali assumono particolare importanza i movimenti del paziente e degli organi al suo interno, come definito dai rapporti numero 50 (del 1993) e 62 (del 1999) dell'ICRU (International Commission on Radiation Units and Measurement).

Le procedure sono in genere complesse e coinvolgono differenti figure professionali, ciascuna con un ben definito ruolo, che comprendono il medico specialista in radioterapia, il fisico sanitario, il tecnico sanitario di radiologia medica, il dosimetrista e l'infermiere professionale, tutte tra loro coordinate. Nella moderna oncologia è inoltre fon- 
damentale che sia presente un'ottimale integrazione, non solo clinica ma anche culturale, tra chi si occupa di radioterapia e gli altri specialisti del settore, al fine di garantire al paziente il miglior approccio multidisciplinare per la risoluzione della sua malattia.

La prescrizione del trattamento avviene secondo un ben precisa sequenza, riassumibile in 6 punti:

1. Valutazione della reale estensione del tumore (stadiazione) con i più opportuni metodi di indagine diagnostica ed, in particolare, mediante la TC, la RM ed ultimamente, la PET.

2. Conoscenza delle caratteristiche e della storia naturale della neoplasia, con particolare riguardo alle possibilità di diffusione locale e regionale, al fine di impostare correttamente i volumi da irradiare (ad esempio, l'inclusione o meno delle aree linfatiche potenzialmente a rischio).

3. Definizione dello scopo della radioterapia (curativo o palliativo).

4. Selezione della modalità di trattamento più idonea, se eslusiva, o in combinazione con altre terapia. Questa scelta può avere un significativo impatto nella scelta dei volumi e delle dosi.

5. Determinazione del volume bersaglio, della dose ottimale e del frazionamento, e della tecnica di irradiazione, tutti parametri che devono essere definiti tenendo conto della sede del tumore, del tipo istologico, delle stadio e di altri fattori relativi alla malattia ed agli organi sani che la circondano.

6. Responsabilità clinica del paziente in trattamento, con la periodica valutazione dello stato del paziente e della malattia, che include l'analisi e la prevenzione degli effetti indesiderati eventualmente indotti, nonché la loro cura.

La radioterapia viene somministrata, negli Stati Uniti ed in altri Paesi Occidentali, in una percentuale variabile tra il 40 ed $50 \%$ dei pazienti che ogni anno si ammalano di tumore. L'Italia ha valori compresi in questo ambito. L'ultima stima sulle attività di radioterapia nel nostro Paese, pubblicata dall'AIRO (Associazione Italiana di Radioterapia Oncologica - Censimento delle strutture e delle attività di radioterapia in Italia, Archimedica Edizioni, Torino, 2003, e periodicamente aggiornata sul sito www.radioterapiaitalia.it) ha riportato per l'anno 2002 che quasi 110.000 pazienti, vale a dire oltre il $42 \%$ dei nuovi casi di tumore 
verificatisi in Italia, sono stati sottoposti a radioterapia nei circa 140 centri pubblici o privati in quell'anno operativi.

\subsection{LA PIANIFICAZIONE DELLA RADIOTERAPIA}

La pianificazione del trattamento radiante è un processo complesso che prevede la determinazione di tutti i parametri considerati ottimali per garantire la miglior cura possibile al malato neoplastico. Per la sua miglior definizione devono essere presi in considerazione, da una parte, i miglioramenti metodologici nell'affrontare la terapia del cancro, con l'integrazione tra le differenti discipline oncologiche e le scienze di base, e dall'altra, lo sviluppo tecnologico che ha consentito di ottenere un elevato livello di qualità delle prestazioni erogate. Gli elementi principali che concorrono alla realizzazione di questo processo riguardano la esatta definizione dei volumi da trattare, la distribuzione della dose, le caratteristiche tecniche del trattamento, il ruolo delle terapie adiuvanti o complementari. Il tutto deve essere inserito in un ben preciso programma di controllo e di verifica della qualità delle procedure eseguite.

\subsection{Il ruolo delle moderne tecniche di imaging in radioterapia}

Lo sviluppo tecnologico nell'ambito della radioterapia consente oggi di praticare tecniche che garantiscono un grado di precisione nella distribuzione della dose al volume bersaglio che non ha precedenti nel passato. Questo progresso si è svolto in maniera sincrona con quello delle metodiche di diagnostica per immagini applicate alla radioterapia, tanto da far assumere a queste un ruolo sempre crescente nella moderna pratica clinica. L'immagine anatomica dei volumi di interesse (il tumore, ma anche gli organi sani ad esso vicini) è quindi la base per la pianificazione del trattamento radiante.

La tomografia computerizzata (TC) è tuttora la modalità di acquisizione dell'immagine di riferimento per la definizione dei piani di trattamento per diverse ragioni: ha una ampia diffusione nei centri ospedalieri, i costi di esecuzione sono accettabili, fornisce immagini di tipo tridimensionale (3D) delle regioni esaminate utili per realizzare tecniche più complesse, non presenta fenomeni di distorsione geometrica 
dell'immagine. Inoltre è in grado di fornire dati precisi sulla densità elettronica su volumi molto piccoli dei tessuti esaminati (pixel), e sono proprio questi valori i dati quantitativi necessari agli attuali sistemi computerizzati per realizzare il calcolo della distribuzione della dose. I moderni apparecchi TC di terza o quarta generazione (TC spirale) consentono inoltre acquisizioni dell'immagine molto rapide ( 40 o più strati in pochi secondi) con riduzione degli artefatti dovuti ai movimenti respiratori o a quelli spontanei di altri organi.

L'uso delle immagini di risonanza magnetica (RM) permette, nei confronti della TC, una migliore definizione del volume bersaglio in alcune specifiche sedi, quale ad esempio il sistema nervoso centrale, facilitando la valutazione della reale estensione del tumore. Esiste inoltre una più ampia possibilità di utilizzare immagini multiplanari, anche se la ricostruzione in $3 \mathrm{D}$ è ora più facilmente praticabile anche con la TC. I problemi principali delle immagini RM sono rappresentati dalla degradazione dovuta alla distorsione geometrica ai margini del campo di vista, e dalla difficoltà di delineare in maniera precisa il contorno del corpo e delle strutture ossee. Inoltre, l'uso della RM per la definizione dei piani di trattamento è limitata dall'assenza di ogni informazione relativa alla densità tessutale. Molte delle limitazioni di una singola modalità di rilevazione dell'immagine possono tuttavia oggi essere superate dalle tecniche di fusione, in particolare tra TC e RM.

Anche gli ultrasuoni sono impiegati in casi selezionati nella definizione del piano di trattamento, specialmente nel caso del tumore della prostata, dove possono guidare l'esecuzione della brachiterapia e consentire il calcolo della dose in tempo reale, mentre procede l'impianto delle sorgenti radioattive.

L'evoluzione delle tecniche di imaging molecolare, prevalentemente basate sulla tomografia ad emissione di positroni (PET) e di singoli fotoni (SPET), e sulla spettroscopia con RM (RMS), possono permettere l'integrazione per la pratica clinica corrente di ulteriori informazioni di tipo biologico, biochimico e funzionali, superando il concetto esclusivamente morfologico rappresentato dalla TC, dalla RM e dalla ecografia.

La PET è correntemente impiegata in oncologia per la stadiazione, il follow-up e, in misura più limitata, nella valutazione della risposta alla terapia. Finora l'imaging fornito dalla PET è stato soprattutto basato sull'impiego di fluoro-desossi-glucosio (FDG) marcato con un radionuclide, il F-18, per la valutazione della captazione di glucosio da parte 
del tessuto neoplastico, a seguito di un aumento di metabolismo e trasporto cellulare. L'uso della PET con FDG può condurre ad una modificazione della stadio di malattia e della strategia terapeutica in oltre il 20\% dei pazienti oncologici (secondo alcuni autori e per alcuni tipi di tumore fino al 40\%). Queste considerazioni sono valide anche per i pazienti che sono in attesa di essere sottoposti a radioterapia e che possono vedere meglio definito il loro volume bersaglio. Oltre alle applicazioni mediante l'uso del FDG, vi sono altre aree di impiego della PET che possono esitare in un significativo miglioramento del trattamento di radioterapia.

L'esame di caratteristiche biochimiche, molecolari e funzionali all'interno del tumore può permettere la definizione di condizioni locali utili al fine di ottimizzare le strategie terapeutiche. La massa del tessuto neoplastico non è quasi mai omogenea rispetto a numerose caratteristiche; queste non necessariamente risultano in una eterogeneità di densità, $\mathrm{o}$, pur manifestandosi con anomalie di densità, non sono attribuibili ad uno specifico processo. Numerose variabili possono infatti determinare specifiche alterazioni del tessuto tumorale non distinguibili attraverso un esame TC, inclusi il grado di vascolarizzazione e di angiogenesi, lo stato di perfusione, l'ossigenazione, il tasso di crescita e il grado di apoptosi, l'espressione di determinati antigeni e recettori. Tutte queste caratteristiche sono parametri utili per definire il grado di aggressività di un tumore e sono correlati alla sua evoluzione. Per tali ragioni la loro caratterizzazione può essere estremamente utile nella pianificazione terapeutica. La rilevazione di aree di tessuto tumorale ipermetaboliche mediante PET con FDG può comportare una migliore definizione della estensione locale e regionale del tumore, vale a dire del cosiddetto Clinical Target Volume (CTV).

In aggiunta a questo, diversi volumi bersaglio possono essere identificati all'interno della stessa massa tumorale, sulla base della captazione di FDG. Aree di elevata captazione possono essere quindi trattate con dosi maggiori di radiazioni rispetto ad aree ipometaboliche della stessa massa. Questo concetto assume una particolare importanza nel caso delle tecniche di modulazione di intensità o della radioterapia con adroni a scanning attivo, che verranno trattate successivamente. Infatti, in tali casi i sottovolumi di ciascuna lesione possono essere irradiati con dosi diverse, nel corso di una singola sessione di trattamento. L'esame PET con FDG può inoltre permettere la definizione di protocolli di trattamento con dosi variabili crescenti sulla base della caratterizza- 
zione biologica del tumore. Inoltre, informazioni biologiche sulla risposta al trattamento con radioterapia e sulla radiosensibilità del tumore, valutata mediante PET con FDG, possono essere impiegate per modificare dopo una settimana o due di trattamento l'iniziale piano di trattamento, estendendo il concetto della cosiddetta terapia adattativi.

Le immagini PET da sole non sono sufficientemente informative dal punto di vista anatomico, per questa ragione la combinazione di PET e TC, basata sull'impiego di tecniche di co-registrazione e fusione, o sull'impiego di scanner ibridi PET/TC possono migliorare la accuratezza del piano di trattamento e della distribuzione della dose. In pazienti con tumore del polmone non a piccole cellule l'uso della PET con FDG permette di escludere la presenza di aree di atelettasia o infezione dal volume bersaglio e di includere invece nello stesso le eventuali lesioni linfonodali individuate e caratterizzate sulla base della captazione di FDG.

Numerosi altri traccianti sono stati recentemente sintetizzati per applicazioni oncologiche. La captazione di due di essi, la metionina e la metil-tirosina, è stata correlata al trasporto, metilazione e incorporazione degli aminoacidi nelle proteine e si ritiene inoltre che la captazione della metionina e della metil-tirosina possa essere considerata indicativa della vitalità cellulare. L'uso della metionina marcata con Carbonio 1 (C-11) e della metil-tirosina, marcata ancora con C-11 o con Iodio 123 per la rilevazione mediante PET o SPET, rispettivamente, può essere più utile dell'impiego del FDG nei tumori cerebrali, poiché il FDG viene normalmente captato da tutto il tessuto cerebrale, a differenza degli aminoacidi, e non solo dal tumore. L'impiego di un altro tracciante, la timidina marcata con F-18, è stato preso in esame per la valutazione della espressione della timidina-chinasi, un enzima chiave per la sintesi dei precursori del DNA e la cui espressione è correlata alla proliferazione cellulare.

Ulteriori importanti risultati sono attesi dall'introduzione della pratica clinica di traccianti radioattivi per la valutazione dell'angiogenesi, della apoptosi e soprattutto dell'ipossia, poiché tutte queste variabili giocano un ruolo cruciale per il successo della radioterapia. Ad esempio, essendo il grado di ossigenazione un fattore critico per il successo della radioterapia, la valutazione di questa variabile può permettere di stimarne l'esito e di identificare i pazienti in cui potrebbe essere utile una terapia radiosenzibilizzante in aggiunta alla radioterapia. Il tracciante più conosciuto per la valutazione dell'ipossia è il fluoro-mi- 
sonidazolo marcato con F-18; anche altri traccianti sono stati studiati ma questo è un campo in piena evoluzione.

Un'altra strategia per la caratterizzazione dei tumori si basa sull'uso di traccianti per la valutazione dell'angiogenesi. I più testati per la valutazione dell'angiogenesi sono rivolti a mettere in evidenza l'espressione dell'alfa(v)beta(3) integrina, una glicoproteina di membrana coinvolta nella migrazione delle cellule endoteliali attivate, la cui espressione aumenta nel corso della neoangiogenesi. Sono stati sviluppati anche metodi per la valutazione dell'apoptosi, prevalentemente impiegati nell'esame dell'esternalizzazione della fosfatidilserina, un risultato della disattivazione/attivazione di altri enzimi intracellulari (traslocasi, floppasi, scramblasi). La possibilità di esaminare l'apoptosi mediante l'annessina $\mathrm{V}$, una proteina che si lega ai fosfolipidi e in particolare con alta affinità per la fosfatidilserina espressa nel corso dell'apoptosi.

La combinazione di RM di spettroscopia (RMS) e imaging RM permette nella pratica clinica di valutare la concentrazione di alcuni metaboliti contenenti protoni, fosforo, fluoro o altri nuclei. Con la spettroscopia dell'idrogeno e del fosforo sono state dimostrate alterazioni rilevanti del metabolismo degli aminoacidi, dei lipidi e di altri composti contenenti fosforo. La fosfocreatina è sovente diminuita nei tumori rispetto alle condizioni normali e relativamente ai tessuti circostanti. Tuttavia tale diminuzione non sembra correlata al grado di malignità. Inoltre le anomalie osservate relativamente allo spettro del fosforo non sono una caratteristica esclusiva delle neoplasie, ma sono presenti anche in altre patologie. Le variazioni di livello dei composti contenenti fosforo sono la norma in corso di radio e chemioterapia. Le variazioni spettroscopiche sono spesso rilevabili prima dei segni morfologici del tumore rappresentati da variazioni di dimensioni e forma dell'organo in esame. Tuttavia risposte con andamento contrastante possono aversi anche indipendentemente dall'evoluzione del tumore. La spettroscopia dei protoni è stata recentemente impiegata con successo nella valutazione della perdita neuronale dopo radioterapia. La RMS è stata recentemente integrata nella pianificazione della terapia per i tumori della prostata.

È evidente che tutte le procedure appena descritte sono più complesse e costose di quelle convenzionali; occorre quindi un'attenta valutazione in termini di rapporto tra costo e beneficio, che deve tenere conto, da un lato, dei maggiori investimenti necessari per realizzare pienamente questi approcci e dall'altro del prevedibile incremento 
delle probabilità di controllo loco-regionale della malattia e della minore incidenza, con il derivante risparmio, di effetti collaterali e di complicanze.

\subsection{L'ottimizzazione del trattamento radioterapico}

La distribuzione ottimale della dose al bersaglio può essere ottenuta con diverse modalità tecniche, quali la combinazione di radiazioni di diversa natura (che nella radioterapia classica sono i raggi $\mathrm{X}$ o gli elettroni) e l'uso di campi singoli o multipli, con complessità crescente che parte dai fasci contrapposti per arrivare alle più complesse tecniche isocentriche, sia statiche che dinamiche mediante opportuni archi di rotazione) od, ancora, alla radioterapia di alta precisione con conformazione della dose basata sulla simulazione virtuale e la ricostruzione 3D. Oltre alla radioterapia dall'esterno, che rappresenta la più frequente modalità di irradiazione (oltre il 95\% dei casi), in rapporto all'accessibilità di particolari sedi anatomiche può poi essere indicata la brachiterapia, realizzabile mediante l'uso di sorgenti radioattive di raggi $\gamma$; questa può rappresentare la sola forma di radioterapia od essere associata a quella a fasci esterni.

In ogni caso, la pianificazione del trattamento prevede, per la localizzazione dei volumi da trattare, l'esecuzione di una specifica procedura, detta di simulazione. Essa precede la fase terapeutica e consente di l'identificazione e la verifica, con la possibilità di ottimizzarli, di tutti gli aspetti geometrici del trattamento, quali l'orientamento dei campi, la dimensione, la forma (che può essere regolare o resa irregolare mediante l'uso di blocchi schermanti o di accessori particolari quali i collimatori multilamellari), ed infine, il posizionamento di punti di reperi sul paziente tali da consentire la riproducibilità della geometria del trattamento per tutte le sedute successive. Convenzionalmente questa procedura viene praticata in una sala del reparto di radioterapia dotata di un particolare apparecchio, chiamato appunto simulatore. Si tratta di un'unità a raggi $\mathrm{X}$ che consente di identificare i campi di irradiazione che saranno poi usati nella fase di trattamento, documentandoli con immagini radioscopiche o radiografie. Il simulatore deve essere analogo agli acceleratori lineari usati per la terapia per quanto attiene le caratteristiche ed i movimenti della testata (o "gantry"), del lettino, e del sistema di collimazione. Invece della sorgente di megavoltaggio il simu- 
latore possiede un tubo radiogeno di tipo diagnostico collegato ad un intensificatore di brillanza, in modo da poter usufruire della radioscopia e consentire in questo modo all'operatore di praticare tutte le procedure adattandosi in tempo reale alle condizioni anatomiche del paziente. Attualmente, i simulatori convenzionali tendono a essere sostituiti dagli apparecchi TC, opportunamente adattati agli scopi della radioterapia per quanto attiene sia il software sia l'hardware (TC-simulatori). In questo modo, è possibile acquisire direttamente una serie di scansioni assiali che consentono una più accurata definizione delle regioni da trattare e della posizione degli organi sani eventualmente compresi nel volume irradiato e predisporre la loro protezione, totale o parziale. La ricostruzione 3D consente poi di incorporare la fase di simulazione con quella del calcolo della distribuzione e realizzare quelle tecniche conformazionale che rappresentano le più moderne espressioni della radioterapia di precisione.

Le procedure di simulazione, sia con apparecchio convenzionale che con TC-simulatore, devono evidentemente essere condotte nelle stesse condizioni geometriche del successivo trattamento. Per questa ragione, è anzitutto necessario garantire l'immobilità del paziente mediante l'uso di diversi sistemi, rappresentati, in rapporto alle parti corporee da trattare, da maschere termoplastiche, schiume di poliuretano, cuscini a espansione, piani inclinati regolabili, reggi-braccia od altri ancora. Strumenti ottici (laser) o elettronici consentono poi di identificare e registrare la posizione del paziente, considerato solidale al lettino, rispetto a un sistema di coordinate spaziali e di poterla quindi agevolmente riprodurre quotidianamente e per tutta la durata della radioterapia.

Alla simulazione fa seguito il calcolo della dose da somministrare. I moderni sistemi sono in grado di utilizzare direttamente le immagini fornite dal simulatore o dalla TC e di tenere conto dei diversi valori di assorbimento delle radiazioni da parte dei tessuti. La prima parte di questa fase fa riferimento alla delineazione dei volumi di interesse, intesi come tumore e tessuti sani, secondo le definizione già citate nell'introduzione a proposito dei rapporti ICRU. I volumi da rappresentare sono in genere più di uno e per ciascuno di questi occorre una accurata rappresentazione grafica. Si tratta quindi una procedura piuttosto complessa, che richiede tempo, ma che fornisce tutti gli elementi per procedere alla esecuzione del piano di cura. Quando questo viene realizzato, il prodotto è rappresentato dall'ottenimento di mappe di di- 
stribuzione della dose che devono essere valutate in modo qualitativo e quantitativo. Particolare attenzione è di solito riservata alla omogeneità della dose, che deve rientrare in criteri di ampiezza prestabiliti, ed al rapporto tra $i$ valori di dose prescritti ed i volumi sui quali questa è distribuita, sia per il tumore che per i tessuti sani. Per questi ultimi esistono dei limiti che non devono essere superati.

Infine, l'ulteriore passaggio è costituito dal trasferimento del piano di cura definitivamente elaborato alle unità di terapia, oggi per lo più rappresentate dagli acceleratori lineari. Queste macchine, che hanno avuto grandi rinnovamenti tecnologici negli ultimi anni, erogano raggi $\mathrm{X}$ di elevata energia (in clinica comprese tra i 4 ed i $25 \mathrm{MV}$ ) od elettroni. Le più moderne sono inoltre dotate di accessori molto utili per aumentare la precisione e l'accuratezza della terapia: lettini robotizzati, collimatori multilamellari, sistemi in grado di rivelare in tempo reale il campo di irradiazione.

Un aspetto fondamentale della radioterapia è rappresentato dalla verifica della qualità del trattamento eseguito, sia per quanto riguarda l'accuratezza del posizionamento del paziente e della geometria dei fasci di irradiazione sia per quanto concerne la dosimetria. Sono ormai generalmente adottate norme di assicurazione della qualità che prevedono venga eseguita con periodicità una serie di controlli non solo sulle macchine e sugli accessori, ma anche sulle procedure e sulla dosimetria, possibile anche in "vivo", cioè sul paziente. Lo scopo è di assicurare l'adeguatezza balistica del trattamento e l'attendibilità dei valori di dose calcolati.

\subsection{LE TECNICHE SPECIALI}

\subsection{Radioterapia 3D-conformazionale}

Si definisce radioterapia 3D-conformazionale (3D-CRT) una tecnica di alta precisione basata sulla definizione volumetrica, tridimensionale del tumore e dell'anatomia degli organi critici. Essa consente di superare i limiti concettuali della radioterapia convenzionale, basata su una anatomia bidimensionale, cioè di superficie, e sul relativo calcolo della dose in un numero limitato di piani. La ricostruzione digitale dei volumi di interesse per la tecnica 3D-CRT è resa possibile dall'acquisizione me- 
diante TC di una serie di sezioni trasversali e contigue della regione da trattare. Particolarmente utili a questo scopo sono le TC spirali, che consentono di ottenere scansioni continue in tempi assai brevi, limitando gli artefatti dovuti al movimento. Queste vengono poi elaborate e ricostruite in 3D da appositi strumenti dedicati ("consolle per treatment planning"), ottenendo quindi il volume corporeo di interesse. Su questa nuova immagine è quindi possibile simulare, ancora in una realtà totalmente virtuale, qualsiasi orientamento dei fasci di irradiazione, ponendosi in una situazione che consente all'operatore di "vedere" il paziente e il bersaglio in modo coincidente con la sorgente di raggi $\mathrm{X}$ della unità di terapia e lungo l'asse del fascio che da essa origina (opzione $\mathrm{BEV}$ - beam's eye-view). In questo modo, si riesce a ottimizzare la geometria del trattamento, scegliendo il numero e l'angolo di incidenza di ogni singolo campo, senza limitazione ai soli piani assiali, e a conformarne in modo assai preciso la forma su quella del bersaglio. Accessori particolari di cui oggi sono forniti i moderni acceleratori lineari, i collimatori multilamellari, consentono poi di realizzare nella pratica questi aggiustamenti. Si tratta di strumenti molto sofisticati e costosi, integrati nella macchina o inseribili al momento necessario, che grazie ad una serie di lamelle, ciascuna governabile in modo indipendente, delle dimensioni comprese tra il centimetro e pochi millimetri, contornano il campo da irradiare rendendo possibile realizzare forme geometriche irregolari e comunque "conformi" al bersaglio.

La 3D-CRT prevede inoltre che il calcolo della dose sia condotto sull'intero volume trattato e non, come avviene convenzionalmente, sulla sola superficie trasversale ricavata dalla definizione del contorno del corpo del paziente. Particolari istogrammi, definiti dose-volume (DVH - Dose Volume Histogram), consentono di confrontare da un punto di vista quantitativo e numerico piani di distribuzione della dose rivali. L'uso dei DVH rappresenta un ulteriore progresso nei confronti della sola valutazione spaziale della distribuzione di dose, ed è utile per individuare il miglior trattamento possibile in termini di probabilità di controllo locale e di complicanze attese.

Il programma di controllo della qualità nella esecuzione delle tecniche di precisione acquista un'importanza determinante. La conformazione in $3 \mathrm{D}$ comporta infatti una riduzione dei margini intorno al volume bersaglio e quindi aumenta il rischio di errore geografico, vale a dire l'omissione di una parte dello stesso con relativo sottodosaggio, se non vengono poste in essere tutte le misure per garantire l'accura- 
tezza balistica del trattamento. Esistono oggi vari strumenti che consentono questi controlli, dai sistemi elettronici di verifica portale (EPID - Electronic Portal Imaging Device), che consentono di estrarre in tempo reale, l'immagine del campo di irradiazione e di confrontarla automaticamente con quella ricavata dalla TC durante la procedura di simulazione, opportunamente ricostruita (DRR - Digitally Reconstructed Radiography), agli apparecchi ottici od optoelettronici o ad altri a raggi $\mathrm{X}$ o ad ultrasuoni, che verificano, anch'essi in tempo reale, la effettiva posizione del paziente e/o del bersaglio nei confronti di quella ideale, consentendo il giusto riallineamento.

Nell'ambito clinico, il razionale su cui si basa l'applicazione della 3D-CRT è quello del miglioramento del controllo locale di malattia. La tecnica consente di ridurre l'esposizione dei tessuti sani e quindi di poter somministrare dosi più elevate, anche del $20-30 \%$, di quelle possibili con tecnica convenzionale, non aumentando così il rischio di complicazioni. Ovviamente la condizione è che possano beneficiarne i pazienti con quei tumori che dimostrano di essere responsivi alla maggior dose somministrata, o che abbiano una scarsa tendenza alla metastatizzazione. Le esperienze cliniche positive riguardano in particolare i tumori della prostata, del polmone, del pancreas e della rinofaringe, dove sono stati raggiunti interessanti risultati, specialmente in termini di riduzione degli effetti collaterali.

\subsection{Radioterapia a intensità modulata}

Mentre la 3D-CRT è somministrata in modo statico, usando campi multipli, fissi e convergenti sul bersaglio anche da piani non complanari, o dinamico, con tecniche isocentriche, è già possibile variare la distribuzione della dose anche agendo su altri parametri, modulando, mediante l'inserimento temporaneo di appositi filtri, l'intensità (o, meglio, la "fluenza") del fascio di irradiazione durante la stessa fase della sua erogazione. Tutto questo è reso possibile dalla integrazione dei sistemi di collimazione multilamellare con i software di gestione dei movimenti della macchina e del calcolo della dose. La radioterapia a intensità modulata (IMRT) rappresenta quindi un'interessante evoluzione della 3DCRT e consente, grazie al completo controllo di tutta la procedura mediante il computer, un ulteriore progresso nella conformazione della dose, creando forme sempre più irregolari, che seguano però la confi- 
gurazione volumetrica del bersaglio da trattare. Le indicazioni cliniche sono pertanto le stesse della 3D-CRT, eventualmente riferite al trattamento di sedi particolarmente critiche per la presenza di organi a rischio molto prossimi al tumore. Si tratta comunque di una tecnica molto complessa, che richiede lunghi tempi di pianificazione, di calcolo e di esecuzione, anche 3 volte superiori a quelli di una normale seduta di radioterapia.

La IMRT è oggi praticata sia con acceleratori lineari opportunamente implementati (collimatori multilamellari "micro", software di gestione automatica dell'erogazione della dose) o con apparecchi dedicati (ad esempio, la, Tomotherapy, uno strumento costituito da una doppia sorgente: una di bassa energia a raggi $\mathrm{X}$ utilizzabile per produrre immagini TC e l'altra di energia elevata, di $6 \mathrm{MV}$, usata a scopo terapeutico) ma richiede procedure di calcolo della dose più complesse, del tipo inverse planning. Si tratta di sistemi che, anziché calcolare la dose basandosi su un'impostazione preliminare dei fasci di radiazioni, attuano il procedimento inverso: viene stabilito una certo livello di dose da somministrare in vari punti del volume da trattare e il sistema fornisce la configurazione geometrica ideale per raggiungere l'obiettivo.

\subsection{Radiochirurgia e radioterapia stereotassica (SRT)}

La "radiochirurgia" fa riferimento a una particolare forma di radioterapia (il termine "chirurgia" è per molti versi improprio) utilizzata per il trattamento di lesioni individuate attraverso metodi stereotassici estremamente accurati. Per le sue caratteristiche, la radiochirurgia consente di erogare un'elevata dose singola (sino 20 Gy ed oltre) a un bersaglio di limitate dimensioni e contemporaneamente di ridurre l'esposizione dei tessuti sani circostanti. Le prime esperienze risalgono agli anni Cinquanta, quando è stata impiegata nel trattamento delle malformazioni arterovenose cerebrali. Negli anni, l'uso è stato esteso alle patologie tumorali, benigne e maligne, primitive o secondarie, del cervello, generalmente di piccole dimensioni. Per l'esecuzione della radiochirurgia cerebrale esistono apparecchi dedicati, chiamati Gamma-Knife, ma analoghi risultati sono ottenibili usando acceleratori lineari dotati di particolari strumenti. La predilezione per l'applicazione alle sedi craniche deriva dalla relativa assenza di movimenti di queste strutture, nonché 
dalla possibilità, attraverso sistemi di immobilizzazione rigidamente applicati all'osso cranico, di ottenere un sistema solidale tra paziente e apparecchiatura ("casco stereotassico") tale da garantire la massima accuratezza. La recente disponibilità di sistemi meno rigidi per l'immobilizzazione e il posizionamento del paziente hanno consentito, in caso di lesioni di dimensioni maggiori o in sedi prossime a strutture radiosensibili, di introdurre la possibilità di frazionare la dose totale in più sedute, riducendo la dose biologica agli organi sani. Da questo concetto deriva la modalità nota come radioterapia stereotassica, analoga alla radiochirurgia, con l'unica differenza legata alla possibilità di praticare più sedute di minore entità, seguendo i principi del frazionamento, quando le caratteristiche della sede lo richiedano.

Il medesimo razionale è stato applicato più recentemente alla terapia di lesioni localizzate al di fuori del cranio, definendo questa modalità ancora come radiochirurgia extracranica (o corporea) se eseguita in una unica frazione o radioterapia stereotassica extracranica se praticata in più sedute. In particolare, nel trattamento di sedi extracraniche l'accuratezza è associata alla necessità di valutare e calcolare i movimenti diretti o indiretti degli organi compresi nel campo di irradiazione. A questo proposito, grande importanza ha il monitoraggio degli atti respiratori, per la loro influenza sulle variazioni di posizione della lesione bersaglio e degli organi in genere. Esistono vari sistemi adatti allo scopo. Tra i più interessanti occorre ricordare quelli opto-elettronici a marcatori passivi. Sulla superficie corporea del paziente vengono posizionati particolari dispositivi la cui posizione è registrata in tempo reale da telecamere sensibili ai raggi infrarossi. Questo consente di valutare in ogni momento la posizione della superficie toracica e di correlare il dato ottenuto con il movimento degli organi interni. L'erogazione della dose può essere coordinata in modo da seguire in tempo reale gli spostamenti del bersaglio e garantirne quindi la copertura.

Tranne che per tale specifico aspetto, il trattamento delle lesioni corporee non differisce concettualmente e tecnicamente da quello delle lesioni cerebrali. Sono infatti impiegati gli stessi principi di localizzazione stereotassica del volume bersaglio, nonché delle strutture critiche da salvaguardare. I trattamenti sono in genere eseguiti medianti acceleratori lineari dotati di particolari collimatori e in grado di erogare fasci multipli non complanari di radiazioni che convergono in modo molto selettivo sul bersaglio, con un notevole risparmio di tessuto sano. La dose totale, molto elevata, può essere frazionata in 2, 3 o 4 sedute, an- 
che se spesso la terapia viene praticata in una sola volta. Il principale vantaggio della radiochirurgia e della radioterapia stereotassica corporea è quindi quello di irradiare sedi e lesioni difficilmente raggiungibili con le metodiche convenzionali, con una durata del ciclo terapeutico molto breve (al massimo una settimana). Gli organi nei quali questa tecnica può essere applicata sono molteplici: polmone, fegato, pancreas, rene, ma anche masse diverse in sede addominale o pelvica. Le dimensioni delle lesioni da trattare devono essere limitate (non oltre $5 \mathrm{~cm}$ nel massimo diametro). La presenza di malattia diffusa, con più sedi interessate, rappresenta un limite all'indicazione. In queste situazioni, purtroppo assai frequenti, deve essere chiaro il ruolo palliativo della metodica, volto alla cura dei sintomi quali il dolore, il sanguinamento, la compressione, ed altri.

Un ulteriore progresso tecnologico è rappresentato da uno speciale acceleratore lineare robotizzato e gestito in modo totalmente automatico (Cyberknife). Esso è in grado di produrre un sottile fascio di radiazioni che, in sequenza, va a colpire il bersaglio, inteso non come una massa singola, ma scomposto a sua volta in una serie di piccoli volumi. Il sistema è anche in grado di compensare alcuni movimenti involontari del paziente o dell'organo trattato. Al momento, le indicazioni all'uso della Cyberknife sono limitate alle lesioni craniche o del massiccio facciale, ma alcune sperimentazioni ne stanno valutando l'efficacia anche in lesioni situate in altre sedi del corpo.

\subsection{Brachiterapia}

Questa tecnica indica l'utilizzazione delle radiazioni a breve distanza o addirittura all'interno del volume bersaglio trattato. Si tratta di una caratteristica peculiare che differenzia la brachiterapia dalle altre metodiche, nelle quali lo schema è rappresentato da un fascio di radiazioni che dall'esterno è indirizzato a colpire il bersaglio. I preparati radioattivi possono essere inseriti in modo invasivo direttamente all'interno del tumore (tecnica interstiziale), o posizionati nelle cavità naturali del corpo (tecnica endocavitaria o endoluminale), oppure posti direttamente sulla superficie cutanea (tecnica di contatto). Naturalmente, la neoplasia deve essere accessibile all'operatore e i suoi contorni ben definiti e facilmente identificabili, in modo da poter situare correttamente le sorgenti dal punto di vista geometrico, requisito essenziale per ottenere 
una distribuzione omogenea della dose. La possibilità di realizzare l'impianto dei cateteri durante una fase operatoria, a cielo aperto, può aumentare ulteriormente l'accuratezza del trattamento (brachiterapia intraoperatoria o perioperatoria).

Lo sviluppo delle conoscenze fisiche nel campo della radioattività ha reso possibile l'uso di sorgenti racchiuse in capsule, sempre più sicure, raffinate e quindi idonee all'impiego clinico. Al radium, ormai abbandonato per le problematiche di radioprotezione che determinava anche a causa della lunghissima emivita, e al cesio, il cui uso è oggi limitato ai soli trattamenti ginecologici, si è oggi frequentemente sostituito l'iridio-192, un emittente $\gamma$ di alta energia $(0,38 \mathrm{MeV})$, di modesta emivita fisica (poco più di 73 giorni), e con un'attività specifica elevata che ne consente la miniaturizzazione in tubi, fili, aghi, spille, sfere e semi dotati di diverse attività. Tra gli altri isotopi recentemente introdotti, un cenno particolare meritano lo iodio-125 ed il palladio-103. Essi possiedono un'energia bassa (con componente $\mathrm{X}$, dell'ordine dei $\mathrm{KeV}$ ) tale da determinare il contenimento quasi totale della dose rilasciata all'interno dell'organo impiantato, con risparmio delle strutture circostanti. La loro elevata attività specifica ne consente la miniaturizzazione sottoforma di piccoli semi, che una volta impiantati, rimangono definitivamente nel paziente (impianto permanente), con un rilascio della dose in tempi molto lunghi, anche di alcuni mesi. La disponibilità di questo tipo di sorgente, accompagnata dall'uso dell'ecografia transrettale per guidare la fase di impianto, ha determinato il grande successo della tecnica di impianto permanente nella cura del carcinoma della prostata in stadio iniziale.

Nel caso degli impianti temporanei, che devono invece essere rimossi dopo il necessario tempo, al diverso livello energetico dei radioisotopi impiegati corrispondono differenti modalità di somministrazione della dose: da quella classica, continua, della durata di qualche giorno (a basso rateo di dose, LDR - Low Dose Rate, con emissione tra 0.4 e 2 Gy per ora), a quella frazionata, della durata di pochi minuti (ad alto rateo di dose, HDR - High Dose Rate, con emissione superiore ai 12 Gy per ora), passando per valori di medio rateo, per la verità poco usati nella clinica. Una recente modalità è quella rappresentata dalla brachiterapia a rateo di dose pulsato (PDR - Pulsed Dose Rate), con esposizione caratterizzata da impulsi della durata di circa 10 minuti, ripetuti in serie, dopo un intervallo di 50 minuti, per 50-60 ore consecutive. 
L'interesse verso la brachiterapia è stato anche favorito dai miglioramenti delle apparecchiature; la protezione degli operatori è stata resa possibile dalle metodiche afterloading, in cui inizialmente si posizionano gli applicatori e solo dopo aver verificato la loro soddisfacente collocazione si introducono le sostanze radioattive, e ancor di più dai proiettori remote afterloading, che consentono l'inserimento a distanza delle sorgenti negli applicatori, eliminando del tutto il rischio di esposizione. Questi apparecchi sono anche in grado di gestire, utilizzando appositi software, in modo corretto dal punto di vista spaziale e temporale il posizionamento automatico delle sorgenti per brachiterapia PDR o HDR. La disponibilità di questi sistemi, accanto a quella di programmi per il calcolo 3D della dose applicabili, in modo analogo alla radioterapia dall'esterno, sull'imaging TC e/o RM degli impianti, hanno inoltre consentito di introdurre metodiche di trattamento di elevata conformazione. Le tecniche ad alto rateo di dose (HDR) consentono poi trattamenti più brevi, dell'ordine di qualche minuto, da ripetersi a distanza di giorni, e fino a un massimo di 6 frazioni, eseguibili anche in regime ambulatoriale. Pertanto, alle indicazioni classiche della brachiterapia (neoplasie ginecologiche, del cavo orale, della cute) se ne sono affiancate di nuove, quali i carcinomi della trachea, dei bronchi e dell'esofago, del retto, delle vie biliari, dei sarcomi, della già citata prostata e di altre sedi tumorali che prima non era agevole trattare a causa del tempo troppo lungo di permanenza delle sorgenti radioattive nella sede della malattia. La brachiterapia sta inoltre occupando un ruolo ben definito per il trattamento delle recidive neoplastiche, anche in territorio già precedentemente irradiato.

\subsection{Radioterapia intraoperatoria}

L'idea di praticare la radioterapia durante l'esecuzione di un intervento chirurgico non è nuova, essendo in modo aneddotico già stata descritta per la prima quasi un secolo fa a proposito di una eviscerazione pelvica. Tuttavia, la moderna radioterapia intraoperatoria (IORT - Intra Operative Radiation Therapy) coincide con l'introduzione degli acceleratori lineari, intorno agli anni Sessanta, e in particolare con l'impiego degli elettroni. Queste radiazioni, come già sottolineato in precedenza, depositano la dose in modo omogeneo, con limitata penetrazione in profondità. Questa caratteristica, che nella 
radioterapia esterna può essere un limite, per la IORT rappresenta un vantaggio, limitando l'esposizione dei tessuti posti oltre il bersaglio reso accessibile chirurgicamente. Normalmente, la IORT è indirizzata al trattamento di tumori localmente avanzati del pancreas, del retto, dello stomaco e di altre sedi che siano resecabili. Nella pratica, seppure in alcuni casi con casistiche numericamente limitate, tale combinazione è stata sperimentata per tutte o quasi le sedi di neoplasia. Il razionale è rappresentato dalla possibilità di sterilizzare un eventuale residuo microscopico o comunque minimo di malattia da una dose singola, molto elevata di radiazioni praticata a cielo aperto, con l'opportunità di "spostare" gli organi sani o almeno parte di essi fuori dal campo. Nel caso di trattamento esclusivo la dose di radioterapia intraoperatoria può raggiungere valori di 20 Gy ed oltre, ma spesso, questa è usata come sovradosaggio con dosi più basse (10-15 Gy). La IORT può essere combinata ad una irradiazione dall'esterno (eventualmente anche con la chemioterapia), che precede o più frequentemente segue la procedura radiochirurgica. Oltre che ai casi di tumore localmente avanzato, ad elevato rischio di recidiva, la IORT sta riscuotendo un grande interesse nel campo del carcinoma mammario in stadio iniziale; esperienze cliniche in corso ne stanno valutando il ruolo come trattamento esclusivo o sovradosaggio dopo chirurgia conservativa, in alternativa alle 6 settimane di radioterapia postoperatoria convenzionale.

Un aspetto tecnico di grande rilevanza per lo sviluppo della IORT è legato alla recente disponibilità di speciali apparecchiature dedicate (acceleratori lineari mobili) che producono solo fasci di elettroni di opportuna energia (tra i 3 ed $12 \mathrm{MeV}$ ) o di tubi radiogeni a bassa energia (dell'ordine di circa $50 \mathrm{KeV}$ ). Entrambi possono essere installati direttamente in sala operatoria senza necessità di particolari modifiche strutturali dell'ambiente ed usati senza rischi di esposizione per gli operatori. L'identificazione del bersaglio e il trattamento avvengono in sala operatoria, con un'ottimale collaborazione tra chirurgo e oncologo radioterapista.Dal punto di vista logistico ed organizzativo si tratta di un enorme vantaggio, in quanto viene evitato l'inconveniente di spostare il paziente dalla sala operatoria al bunker di trattamento e viceversa, che prolungando il tempo dell'intervento, la durata dell'anestesia e aumentando la complessità procedurale, ha rappresentato il maggior ostacolo alla diffusione delle tecniche intraoperatorie. 


\subsection{Radioterapia con adroni}

Nell'ambito delle tecniche di precisione, la radioterapia con adroni o adroterapia sta acquisendo un ruolo emergente. Si tratta dell'impiego, in sostituzione dei classici raggi $\mathrm{X}$ o $\gamma$, di radiazioni corpuscolari, subatomiche, quali protoni, neutroni e ioni, genericamente definibili come adroni. Per le loro caratteristiche, i protoni e gli ioni hanno la capacità di depositare la propria energia, e quindi la dose in modo assai definito; interagiscono poco lungo il corridoio di entrata, rilasciano tutta la loro energia in un tratto assai limitato, il cosiddetto picco di Bragg, e non proseguono oltre il bersaglio. Sono quindi dotati di un'elevatissima selettività balistica, il che li rende particolarmente adatti a trattare lesioni situate in stretta prossimità con organi critici, a rischio di essere danneggiati. Nel confronto tra distribuzioni di dose ottenibili con IMRT, considerata il miglior standard terapeutico disponibile con i raggi $\mathrm{X}$, e adroterapia con protoni, in varie sedi tumorali, si può quasi sempre osservare che, mentre la copertura del bersaglio è ottimale con entrambe le tecniche, i protoni consentono invece di ridurre in maniera molto significativa la dose agli organi sani circostanti.

I trattamenti con adroni risalgono a molti anni fa, essendo stati praticati sin dagli anni '50 in centri di fisica, a livello sperimentale, ma solo dagli inizi degli anni '90 sono diventati una pratica clinica in alcuni centri specialistici. I pazienti trattati nel mondo con adroni sono complessivamente più di 60 mila, dei quali la maggior parte con protoni (oltre 40 mila). La patologia più frequentemente trattata è quella relativa al melanoma dell'uvea. I risultati ottenuti sono da considerarsi eccezionali: oltre il $95 \%$ dei pazienti con controllo locale della malattia, il $90 \%$ con preservazione dell'occhio, e, di questi, la metà mantenendo un'adeguata acuità visiva. Anche altri tumori rari, quali i cordomi, i condrosarcomi e i meningiomi della base cranica, hanno beneficiato di questa terapia. Recentemente stanno emergendo nuove indicazioni, in particolare per quei tipi di tumore nei quali i livelli di dose elevati hanno dimostrato di essere efficaci nell'incrementare il controllo locale (prostata e polmone, ad esempio). Per quanto attiene gli ioni, dei quali l'unico impiegato in clinica è quello del carbonio, oltre alla già citata capacità balistica, posseggono un'efficacia biologica elevata, particolarmente idonea a curare i tumori cosiddetti radioresistenti, aprendo quindi nuove prospettive per il trattamento di lesioni che tradizionalmente erano escluse dalla radioterapia. 
Lo sviluppo dell'adroterapia è ancora limitato, fondamentalmente per gli elevati costi, dovuti ai grandi sincrotroni o ciclotroni necessari per accelerare le particelle adroniche sino a valori di energia dell'ordine dei $60-450 \mathrm{MeV}$. Tuttavia molte iniziative sono in fase di realizzazione. I centri più noti che già svolgono una attività clinica corrente sono negli Stati Uniti (Boston e Loma Linda, in California) e in Giappone (Chiba, Tsukuba, Hyogo). In Europa sono attivi centri in Svizzera, Francia e Germania. In Italia è iniziata l'attività clinica nei laboratori di fisica di Catania limitatamente al melanoma oculare; un importante progetto di carattere nazionale (CNAO, Centro Nazionale di Adroterapia Oncologica) è in corso di attuazione a Pavia, con previsione di concludere la costruzione del centro entro la fine del 2007 e quindi di iniziare una regolare attività clinica sia con protoni che con ioni carbonio. 
